\title{
EDITORIAL
}

\section{The secret life of steroids in asthma}

\author{
P.J. Sterk*, C.Y. Yick* and A.M. Slats ${ }^{\#}$
}

$\Delta$ sthma is an inflammatory disease. Who is still questioning this paradigm? In the Global Initiative for Asthma (GINA) guidelines the disease is defined as a chronic inflammatory disorder of the airways [1]. The evidence for this is overwhelming, and comes from observational studies as well as experimental models. The proof of the pudding has been provided by numerous studies with anti-inflammatory interventions. Ever since the early 1950s [2], the efficacy of both short courses and maintenance treatment with corticosteroids in asthma has been beyond any discussion [1]. Hence, the evidence that inflammation is causally involved in the pathophysiology of asthma nicely meets Koch's postulations.

Therefore, it is no surprise that ever since this key mechanism of the disease was identified, many academic and industrial groups rushed to develop the most selective and potent antiinflammatory compounds for suppression, prevention or perhaps even cure of the disease. Remarkably, during the past 20 yrs the development of new anti-inflammatory therapies for asthma has been less successful than was hoped and expected [3]. Can't we identify the responsible inflammatory targets? Should such targets be combined? Certainly, this cannot be excluded and deserves an even more intensive search. But, is it not frustrating to see our neighbour rheumatologists successfully testing and implementing one novel anti-inflammatory after the other [4]?

Of course, asthma is a complex disease, perhaps even more complex than arthritis. But what does that mean? If anything, that must be the key point. Complexity requires multidimensional approaches. Should we keep on addressing asthma as reductionists, or does the disease require a more integrative approach, by taking the elaborate dynamics of the organ and the organism into account rather than singling out molecular pathways? It seems that the puzzle of asthma demands the latter. Fortunately, we are moving on to familiar ground here, because this is physiology (or "systems biology" by today's nomenclature). Physiology has always been very strong in respiratory medicine, and we must take advantage of that. MACKLEM [5] may well be right in predicting that deep understanding of the adaptable, self-organised order of biological systems in health and disease will create the next biomedical revolution.

\footnotetext{
*Academic Medical Centre, University of Amsterdam and ${ }^{\text {\# }}$ Sint Lucas Andreas Hospital Amsterdam, The Netherlands.
}

STATEMENT OF INTEREST: None declared.

CORRESPONDENCE: P.J. Sterk, Dept Respiratory Medicine, F5-259. Academic Medical Centre, University of Amsterdam, P.0. Box 22700, NL-1100 DE Amsterdam, The Netherlands. Fax: 31 205669001.E-mail: p.j.sterk@amc.nl
Consequently, is asthma more than inflammation? In principle, asthma is a disease of (variable) airflow limitation. That is to say, episodes of too much narrowing with too little or too short re-widening of the airways. Usually, this is referred to as airway hyperresponsiveness, even though the impaired physiological ability to adequately dilate narrowed airways is not often included in this term (yet). Over the past few years, airway hyperresponsiveness has often been considered as a surrogate measure of airways inflammation [6]. Inflammation was thereby put in the driver seat, with functional abnormalities of the airways merely as the consequence. Well, here we are in 2008. The cracks of this paradigm may best be illustrated by the latest revision of GINA [1]. Whereas the previous definition of asthma in the 2004 GINA report [7] used the phrase "chronic inflammation is causing an associated increase in airway hyperresponsiveness", the current 2007 update stepped back by saying "chronic inflammation is associated with airway hyperresponsiveness" [1]. This essential change of wording did not drop from the skies.

Animal models of asthma warned us that airway inflammation and airway hyperresponsiveness do not correspond well enough $[8,9]$. And indeed, targeted interventions in patients with asthma, e.g. by anti-tumour necrosis factor- $\alpha$ [10] or antiimmunoglobulin E [11], have shown benefits on either hyperresponsiveness or inflammation, but not on both. Let's face it, there is a discrepancy between abnormal function and inflammation of the airways in asthma, at least when considering airway inflammation as measured by inflammatory cell counts in sputum or the lamina propria of bronchial biopsy specimens. The latter does not cover inflammation in its broad sense, which has to include other compartments of the airway wall, inflammatory cell activity and other features, such as oedema, hyperaemia and perhaps even longer term structural changes. But even the latter, often referred to as airway remodelling, does not match well with airway hyperresponsiveness $[12,13]$. This means that, before closing the debate on the relationship between airway inflammation and hyperresponsiveness, we need to reconsider the appropriate measures of both outcomes. For instance, inflammation within the airway smooth muscle layer in asthma [14] does correspond with airway hyperresponsiveness [15]. Measures of hyperresponsiveness other than just provocative concentration causing a $20 \%$ fall in forced expiratory volume in one second (such as deep inspiration-induced bronchodilation and the maximal degree of airway narrowing) do appear to be associated with inflammatory markers within the airway wall $[15,16]$. Therefore, more than the standard measures are required when investigating the potential mechanisms (and treatment) of variable airflow limitation. 
This is exactly the angle that LAKSER et al. [17] chose in the current issue of the European Respiratory Journal. Here one recognises a physiological approach. Their study is based on the notion that the most potent, endogenous bronchodilating mechanism of humans in vivo appears to be grossly impaired in asthma. This refers to bronchodilation following a simple, deep sigh as observed in normal subjects after encountering a bronchoconstrictor stimulus [18]. The mechanism of deep inspiration-induced bronchodilation is likely to be related to the observation that airway smooth muscle cells can adapt to mechanical changes in their microenvironment [19]. Peribronchial pressures vary during inspiration and expiration, which imposes cyclic force fluctuations on the airway wall. These force fluctuations can periodically stretch airway smooth muscle, which reduces the development of active force as well as the stiffness of the muscle [19]. This will impede smooth muscle shortening and promote relaxation of precontracted muscle. In asthma in vivo, such endogenous protection largely fails, which may be due to mechanisms related to smooth muscle itself, its environment or both [20].

LAKSER et al. [17] reasoned that if this is the key abnormality of airway function in asthma (as opposed to airway inflammation per se), the re-lengthening of smooth muscle by force fluctuations should be steroid sensitive. The hypothesis was that steroids inactivate a potential suppressor of force fluctuation-induced relengthening, p38 mitogen-activated protein kinase (MAPK) [21], by increasing the expression of MAPK phosphatase-1 (MKP-1). This inactivates p38 MAPK, thereby enhancing the stretch of precontracted airway smooth muscle by periodic strain. The data show that [17], first, incubation of isotonicly shortened canine tracheal smooth muscle strips with dexamethasone prior to and during force fluctuations significantly augment muscle relengthening. Secondly, dexamethasone increases the expression of MKP-1 in cultured canine tracheal smooth muscle cells, which is accompanied by a decrease in phosphorylation of a downstream target of p38 MAPK, heat shock protein 27 [17]. In short, steroids promote the re-lengthening of smooth muscle by cyclic stretch and also inhibit a suppressor signalling pathway of this, fitting in with each other.

The strength of the study is its functional approach. The value of this cannot easily be underestimated. There is huge progress in the cell biological research of airway smooth muscle, particularly with regard to its phenotype (contractile, secretory and proliferative) and its close interaction with surrounding matrix and neighbouring (inflammatory) cells [22]. However, the vital step here is the link with smooth muscle function and, in particular, the dynamics of it, based on the rapid progress in understanding the biophysics of airway narrowing [19, 20, 23]. LAKSER et al. [17] mimicked the physiological oscillatory conditions of airway smooth muscle, which is critical. They directly assessed the effects of the major anti-asthma drug on what is increasingly believed to be the fundamental functional characteristic that distinguishes asthma from controls; namely, the mechanical changes of the muscle secondary to force fluctuations that occur during normal breathing and after taking an occasional deep breath. This is just what happens at the organ level in all humans.

However, the study also has significant limitations that need to be taken into account [17]. First, this is canine and not human smooth muscle. Secondly, this is not smooth muscle obtained from asthmatics or from a model of asthma. Hence, all the study demonstrates is that re-lengthening by force fluctuations of "normal" muscle strips can be increased by steroids [17]. In fact, the re-lengthening was initiated by steroids because it was absent in the control strips. Therefore, the study does not answer the question as to whether impaired re-lengthening of smooth muscle from asthmatics can also be restored by steroids. Notably, impaired re-lengthening of smooth muscle from patients with asthma in vitro still needs to be established. This seems to be a priority for the field, regardless of whether it is a primary or a secondary abnormality $[19,20]$. Finally, the study does not show a causal relationship or a statistical association between the changes in signalling pathways and those in muscle re-lengthening. The observations are therefore hypothesis generating, rather than hypothesis testing.

What hypotheses can be generated from this study? Most importantly, the study raises the possibility that steroids restore airway function in asthma through direct effects on airway smooth muscle rather than by suppressing inflammation. This suggests that we confused a steroid-sensitive disease with an inflammatory disease! Steroids are not anti-inflammatory per se [24]. Steroids affect gene transcription in virtually any cell type, not only in inflammatory cells, but also in airway resident cells, including airway smooth muscle [25]. The responsible signalling pathways are not fully understood, and could not only be related to transcriptional and posttranscriptional inhibition of MKP-1 and calcium-mobilising second messengers [26], but also to altered transcription of proteins that are responsible for airway smooth muscle phenotype, including contractile elements, cytoskeleton, cell surface molecules, and cytokines or mediators with autocrine function [23, 27]. This will inevitably influence contractile, secretory and proliferative activity. Now it is time to delineate which of these pathways is most appropriate to enhance stretch-induced relaxation of airway smooth muscle in asthma. The physiologists have already taken up the gauntlet [19]. It requires directly linking the dynamics of airway narrowing in asthmatics in vivo to phenotypic markers of airway smooth muscle [28], which can now be expanded by using highdimensional gene expression profiles. If this does not demand an integrative approach, what does?

Meanwhile, steroids will continue to be effective in patients with asthma. They do have anti-inflammatory effects [29] and they do restore the endogenous bronchodilation by a deep sigh in patients with asthma [30]. The secret is which of these benefits the patients most? Or alternatively, which of these is the critically impaired activity by steroids that troubles the severe asthma patients with steroid resistance most [31]? Is it the lack of anti-inflammatory effects or the inadequate normalisation of airway smooth muscle dynamics? The hypothesis is out that it is the latter!

\section{REFERENCES}

1 Bateman ED, Hurd SS, Barnes PJ, et al. Global strategy for asthma management and prevention: GINA executive summary. Eur Respir J 2008; 31: 143-178.

2 Harris MS. Cortisone in treatment of bronchial asthma. Calif Med 1951; 75: 85-88. 
3 Hanania NA. Targeting airway inflammation in asthma: current and future therapies. Chest 2008; 133: 989-998.

4 Voulgari PV. Emerging drugs for rheumatoid arthritis. Expert Opin Emerg Drugs 2008; 13: 175-196.

5 Macklem PT. Emergent phenomena and the secrets of life. J Appl Physiol 2008; 104: 1844-1846.

6 van Schoor J, Joos GF, Pauwels RA. Indirect bronchial hyperresponsiveness in asthma: mechanisms, pharmacology and implications for clinical research. Eur Respir J 2000; 16: 514-533.

7 Global Initiative for Asthma. 2004 Update: Workshop Report, Global Strategy for Asthma Management and Prevention. Www.ginasthma.org/Guidelineitem.asp? $11=$ $2 \& 12=1 \&$ int $I d=87$.

8 Palmans E, Kips JC, Pauwels RA. Prolonged allergen exposure induces structural airway changes in sensitized rats. Am J Respir Crit Care Med 2000; 161: 627-635.

9 Southam DS, Ellis R, Wattie J, Inman MD. Components of airway hyperresponsiveness and their associations with inflammation and remodeling in mice. J Allergy Clin Immunol 2007; 119: 848-854.

10 Berry MA, Hargadon B, Shelley M, et al. Evidence of role of tumor necrosis factor- $\alpha$ in refractory asthma. $N$ Engl J Med 2006; 354: 697-708.

11 Van Rensen ELJ, Evertse CE, van Schadewijk AAM, et al. Eosinophils in bronchial mucosa of asthmatics after allergen challenge: effect of anti-IgE treatment. Allergy 2008; (In press).

12 Mauad T, Bel EH, Sterk PJ. Asthma therapy and airway remodeling. J Allergy Clin Immunol 2007; 120: 997-1009.

13 Siddiqui S, Mistry V, Doe C, et al. Airway hyperresponsiveness is dissociated from airway wall structural remodelling. J Allergy Clin Immunol 2008; 122: 335-341.

14 Brightling C, Bradding P, Symon FA, Holgate ST, Wardlaw AJ, Pavord ID. Mast cell infiltration of airway smooth muscle in asthma. N Engl J Med 2002; 346: 1699-1705.

15 Slats AM, Janssen K, van Schadewijk A, et al. Bronchial inflammation and airway responses to deep inspiration in asthma and chronic obstructive pulmonary disease. Am J Respir Crit Care Med 2007; 176: 121-128.

16 Möller GM, Overbeek SE, van Helden-Meeuwsen CG, Hoogsteden HC, Bogaard JM. Eosinophils in the bronchial mucosa in relation to methacholine dose-response curves in atopic asthma. J Appl Physiol 1999; 86: 1352-1356.

17 Lakser OJ, Dowell ML, Hoyte FL, et al. Steroids augment relengthening of contracted airway smooth muscle: potential additional mechanism of benefit in asthma. Eur Respir J 2008; 32: 1224-1230.
18 Skloot G, Permutt S, Togias A. Airway hyperresponsiveness in asthma: a problem of limited smooth muscle relaxation with inspiration. J Clin Invest 1995; 96: 2393-2403.

19 Fredberg JJ. Bronchospasm and its biophysical basis in airway smooth muscle. Respir Res 2004; 5: 2.

20 An SS, Bai TR, Bates JL, et al. Airway smooth muscle dynamics: a common pathway of airway obstruction in asthma. Eur Repir J 2007; 29: 834-860.

21 Lakser OJ, Lindeman RP, Fredberg JJ. Inhibition of the p38 MAP kinase pathway destabalizes smooth muscle length during physiological loading. Am J Physiol Lung Cell Mol Physiol 2002; 282: L1117-L1121.

22 Tliba O, Amrani Y, Panettieri RA. Is airway smooth muscle the "missing link" modulating airway inflammation in asthma? Chest 2008; 133: 236-242.

23 Halayko AJ, Tran T, Ji SY, Jamasaki A, Gosens R. Airway smooth muscle phenotype and function: interactions with current asthma therapies. Curr Drug Targets 2006; 7: 525-540.

24 Barnes PJ. Corticosteroid effects on cell signalling. Eur Respir J 2006; 27: 413-426.

25 Hakonarson H, Halapi E, Whelan R, Gulcher J, Stefansson K, Grunstein MM. Association between IL-1 $\beta$ / TNF- $\alpha$ induced glucocorticoid-sensitive changes in multiple gene expression and altered responsiveness in airway smooth muscle. Am J Respir Cell Mol Biol 2002; 25: 761-771.

26 Kang BN, Jude JA, Panettieri RA, Walseth TF, Kannan MS. Glucocorticoid regulation of CD38 expression in human airway smooth muscle cells: role of dual specificity phosphatase 1. Am J Physiol Cell Mol Physiol 2008; 295: L186-L193.

27 Zuyderduyn S, Sukkar MB, Dhaliwal S, Burgess JK. Treating asthma means treating airway smooth muscle cells. Eur Respir J 2008; 32: 265-274.

28 Slats AM, Janssen K, van Schadewijk A, et al. Expression of smooth muscle and extracellular matrix proteins in relation to airway function in asthma. J Allergy Clin Immunol 2008; 121: 1196-1202.

29 Laitinen LA, Laitinen A, Haahtela T. A comparative study of the effects of an inhaled corticosteroid, budesonide, and a $\beta 2$ agonist, terbutaline, on airway inflammation in newly diagnosed asthma: a randomized, double-blind, parallelgroup controlled trial. J Allergy Clin Immunol 1992; 90: 32-42.

30 Bel EH, Timmers MC, Hermans J, Dijkman JH, Sterk PJ. The long-term effects of nedocromil sodium and beclomethasone dipropionate on bronchial responsiveness to methacholine in non-atopic asthmatic subjects. Am Rev Respir Dis 1990; 141: 21-28.

31 Adcock IM, Barnes PJ. Molecular mechanisms of corticosteroid resistance. Chest 2008; 134: 394-401. 